\title{
Auditory Recognition of Digit-in-Noise under Unaided and Aided Conditions in Moderate and Severe Sensorineural Hearing Loss
}

\author{
Mina Aghasoleimani, Hamid Jalilvand, Mohammad Ebrahim Mahdavi, and Roghayeh Ahmadi \\ Department of Audiology, School of Rehabilitation, Shahid Beheshti University of Medical Sciences, Tehran, Iran
}

Received February 22, 2020

Revised May 29, 2020

Accepted August 25, 2020
Background and Objectives: The speech-in-noise test is typically performed using an audiometer. The results of the digit-in-noise recognition (DIN) test may be influenced by the flat frequency response of free-field audiometry and frequency of the hearing aid fit based on fitting rationale. This study aims to investigate the DIN test in unaided and aided conditions. Subjects and Methods: Thirty four adults with moderate and severe sensorineural hearing loss (SNHL) participated in the study. The signal-to-noise ratio (SNR) for $50 \%$ of the DIN test was obtained in the following two conditions: 1) the unaided condition, performed using an audiometer in a free field; and 2) aided condition, performed using a hearing aid with an unvented individual earmold that was fitted based on NAL-NL2. Results: There was a statistically significant elevation in the mean SNR for the severe SNHL group in both test conditions when compared with that of the moderate SNHL group. In both groups, the SNR for the aided condition was significantly lower than that of the unaided condition. Conclusions: Speech recognition in hearing-impaired patients can be realized by fitting hearing aids based on evidence-based fitting rationale rather than by measuring it using free-field audiometry measurement that is utilized in a routine clinic setup.

J Audiol Otol 2021;25(2):72-79

KEY WORDS: Sensorineural hearing loss · Hearing aid · Digit-in-noise recognition test Speech intelligibility $\cdot$ Degree of hearing loss.

\section{Introduction}

People with sensorineural hearing loss (SNHL) have difficulty in understanding sounds because of the inaudibility that occurs as a result of hearing loss, especially when listening to speech with a competing background noise. However, although the speech could not be understood because of the inaudibility of speech signals, it has been known that hearingimpaired subjects extract less speech information than healthy subjects for the same level of audibility [1]. Thus, this fact proves that some part of this problem is due to the lower audibility of speech signals [1], especially the high-frequency cues of speech that are most important for understanding speech in noisy environments [2]. The other main part of the issue is

This is an Open Access article distributed under the terms of the Creative Commons Attribution Non-Commercial License (https://creativecommons.org/licenses/by-nc/4.0/) which permits unrestricted non-commercial use, distribution, and reproduction in any medium, provided the original work is properly cited. explained by the inability of the cochlea to resolve the components of complex sounds spectrally due to reduced frequency selectivity; broader auditory filters of the impaired cochlea, which allow passage of more background noise from these filters; and finally, inability to separate speech components from background noise [1].

It is well-known that compared with pure-tone threshold audiometry, which evaluates the subject's hearing acuity, speechin-noise tests can demonstrate the subject's performance in the presence of noise [3-5]. There are various speech-in-noise tests with different approaches that have a variety of contexts and contents; there are also different perspectives on measuring the magnitude of speech intelligibility in the presence of noise. Currently, the digit-in-noise recognition (DIN) test is used because it is easy to standardize and requires minimal language skills because it uses simple digits [6,7]. In addition to low redundancy, it is thought that it is independent from top-down processing and strongly depends on bottom-up processing 
(auditory peripheral function, i.e., audibility of speech signals) [8]. Additionally, no learning effect, low measurement error, high validity, and independence from aging-related cognitive status are other advantages of the DIN test [8-11]. Thus, it could be used as a test to evaluate peripheral auditory processing or verify the effect of a fitted hearing aid on the subject's performance in the presence of noise based on the benefit of the compensated audibility that is provided by hearing aid amplification. Highly correlated with the results of pure-tone threshold audiometry, the DIN test consists of the digit triplets presented in competing background noise, which is expressed as a signal-to-noise ratio (SNR) $[9,10]$. The lower the SNR, the better the performance a subject exhibits in terms of speech recognition in the presence of noise [7]. Thus, by determining the SNR, we can evaluate hearing performance in the presence of noise, especially when amplifying sounds using a hearing aid.

In clinical practice, the subject's auditory performance in real-life situations is predicted from her or his results on the standard audiological test battery. It is specifically assumed that the speech recognition of hearing-impaired subject evaluated using headphones or free-field testing (i.e., unaided condition) is similar to his or her speech recognition with the best-fit hearing aid in the sound field (aided condition). During the first patient's visit, inaccessibility to an individually fitted hearing aid with a special gain frequency response based on evidence-based rationale, as well as with an individualized ear coupling system (e.g., earmold, venting, and sound bore), speech-in-noise tests are routinely performed using a clinical audiometer via headphones or free-field speaker rather than with a hearing aid fit based on hearing loss configuration. Using prescribed amplification in order to provide speech understanding for people with different degrees of hearing loss, signal audibility in different frequency regions is most important [12]. As mentioned in Harvey Dillon's book, Hearing Aid (p.278), since a subject's performance in the presence of noise might be affected by the frequency response that he or she receives, there may be a concern about the different performance when testing for the flat frequency response of the audiometer and individually shaped gain frequency response of a hearing aid fit based on evidence-based fitting rationale [12]. In fact, a hearing aid not only amplifies the sounds, but also reshapes the speech spectrum relative to the flat frequency response of the audiometer [12]. It reshapes the speech spectrum according to the amplification targets based on the patient's hearing loss configuration. If there would be a difference between the two conditions (unaided vs. aided), then counseling for hearing aid candidacy and deciding on hearing aid fit based on the results given by an audiometer may not be accurate and effec- tive. The gain frequency response of a hearing aid might affect the subject's speech performance in the presence of noise due to difference in audibility over the frequency range [12]. For instance, compared with a hearing aid fit based on evidence-based fitting rationale [e.g., National Acoustic Laboratories-Nonlinear, Version 2 (NAL-NL2)], the frequency response of sounds presented using an audiometer via a speaker is totally different. For a patient with more severe SNHL in the high-frequency range, the former may provide an emphasis for high frequencies, but the latter has flat frequency response [12]. Unfortunately, there are not enough studies that have comprehensively investigated this issue. Although the authors know of just one study [13] that compares the results under unaided and aided conditions, it used a word recognition measure in patients with active middle implants rather than a DIN measure in patients with an air conduction hearing aid. However, there is not any study to evaluate the Persian version on DIN among various hearing loss degrees. Therefore, our main study aim was to investigate the subjects' performance in the DIN test in two situations (unaided condition, testing through the use of an audiometer with a speaker in the free field; and aided condition, testing through the use of a hearing aid fit on the basis of NAL-NL2) and compare the results. In addition, we evaluated two groups of subjects with two degrees of hearing loss, moderate and severe SNHL, to investigate the effects of degree of hearing loss.

\section{Subjects and Methods}

\section{Subjects}

A total of 34 adults with hearing loss (19 males and 15 females) participated in the study. Considering the effects of acclimatization on speech recognition performance [12], those with at least 2 years of experience of using hearing aid amplification were recruited. The average usage of hearing aid was at least 10 hours a day based on the hearing aid data logging. Evaluations were performed unilaterally (19 right and 15 left ears). The two study groups were as follows: 1) G1-moderate SNHL group; 2) G2-severe SNHL group.

Each study group had 17 participants. Based on the classification of degree of hearing loss calculated from a pure-tone threshold average (PTA) for 500, 1,000, and 2,000 Hz, defined in the Handbook of Clinical Audiology [14], the first group (G1) comprised 17 subjects with moderate SNHL with a PTA of $46.56 \pm 4.65 \mathrm{~dB} H L$ (ranging from 38.33 to $56.67 \mathrm{~dB} \mathrm{HL}$ ); the second group (G2) comprised 17 subjects with severe SNHL with a PTA of $70.68 \pm 3.06 \mathrm{~dB}$ HL (ranging from 65 to $76.67 \mathrm{~dB}$ HL) (Fig. 1).

The mean age of the study participants was $62.4 \pm 5.2$ years 
and $65.7 \pm 4.8$ years in the moderate and severe SNHL groups, respectively, which was not significantly different. The range of hearing loss in G1 was from mild to moderate at low frequencies that extended to severe at high frequencies, whereas the hearing loss for $\mathrm{G} 2$ was from moderate to severe at low frequencies and severe to profound at high frequencies (Fig. 1).

\section{Procedure}

Despite the fact that DIN test results are typically independent of cognitive status $[8,10]$, each participant was evaluated using the Mini-Mental State Examination (MMSE) in order to control for the potential effects of cognitive status. The cutoff score was 23 or higher on the MMSE [15]. After obtaining signed consent, basic audiological measurements, such as otoscopy evaluation, immittance audiometry, pure-tone audiometry, and speech audiometry, were performed. After that, the ear impression was taken to make an earmold for each participant. This session lasted for 1.5 hours, on average, for each participant.

In the next session, which was the hearing aid fitting, real ear measurement (REM) and speech-in-noise recognition measurements were performed. The Siemens BTE Hearing Aid Motion 301 P BTE (Siemens Hearing Instruments Inc., Erlangen, Germany) was used in the study. For REM, the FP35 Hearing Aid Analyzer was used (Frye Electronics Co., Beaverton, OR, USA). Farsi auditory recognition of DIN (FARDIN) measurements were randomly performed in unaided and aided conditions. This session lasted for 1.5 hours on average. A resting period (approximately 15-30 minutes) was provided

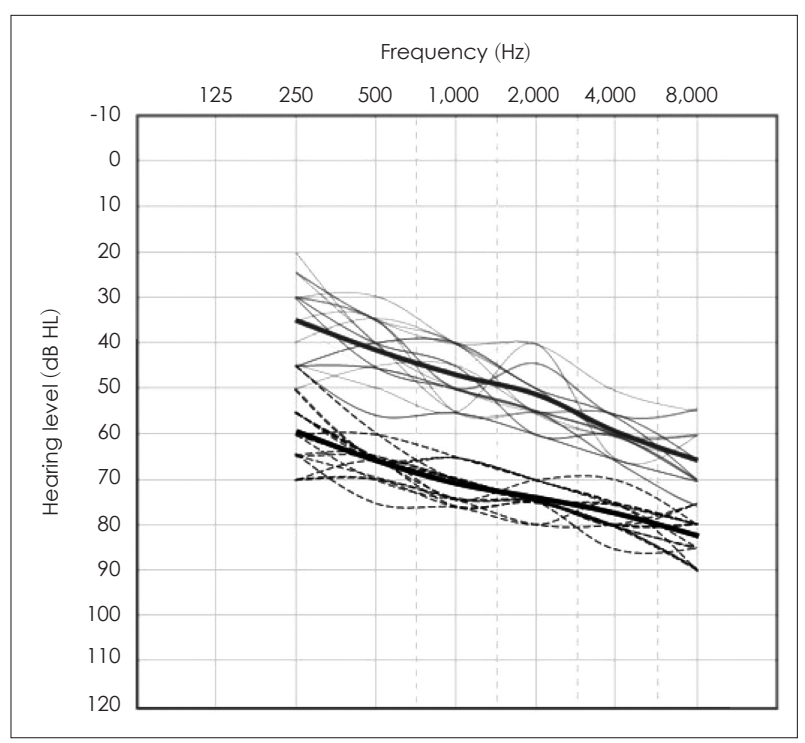

Fig. 1. The individual audiograms of $\mathrm{G} 1$ (moderate $\mathrm{SNHL}$ ) and G2 (severe SNHL) are shown with grey and black dotted curves, respectively. The mean audiograms for each group are shown by their dedicated bold curves. SNHL: sensorineural hearing loss. during testing if necessary. During all measurements, the other ear (untested ear) was occluded by the participant's own ear impression. All the used procedures, methods, and tests were approved by the ethical committee of Shahid Beheshti University of Medical Sciences (Approval No. 139909_03).

\section{Hearing aid fitting}

In the present study, the same BTE Hearing Aid with an unvented individual hard canal earmold (the Siemens Motion 301 P BTE, Siemens Hearing Instruments Inc.) was fitted to all subjects to provide similar amplification conditions. The hearing aid was fitted based on the NAL-NL2 rationale according to SIFIT (v6.11) fitting software. Hearing aid amplification was adjusted based on NAL-NL2 targets for inputs of 50, 65, and $80 \mathrm{~dB}$ SPL. The Real Ear Aided Responses were measured using the broadband stimulus, Digital Speech, at levels of 50, 65 , and $80 \mathrm{~dB}$ SPL to represent soft, medium, and high inputs, respectively. All amplification measures had to be within the range of $\pm 3 \mathrm{~dB}$ of the NAL-NL2 targets for the mentioned inputs. In addition, the maximum pressure output was set according to NAL-NL2 targets for warble pure-tone input at $90 \mathrm{~dB}$ SPL. The default amplitude compression settings were used. An omnidirectional microphone was set for all participants, and other digital signal processing algorithms (such as Sound Smoothing, eWind Screen, and Feedback Stopper) were inactivated. Based on the protocol introduced by Shahid Beheshti University of Medical Sciences (BUMS) for fitting the digital processing hearing aid, each participant was evaluated using the calibrated version of the Ling-6 sounds for evaluation of aided detection thresholds [16-18]. Despite the fact that DIN test was accomplished in suprathreshold level, the hearing aid was fitted so that the Ling-6 sounds were audible and discriminative in the level of $30 \mathrm{~dB}$ HL to provide equal audibility for all subjects.

\section{Test materials}

\section{DIN test}

All sound field testing was performed in a sound attenuated booth that met ANSI standards for sound attenuation [19]. In this study, the Persian version of the DIN test, FARDIN, was used to measure the participant's speech perception performance in the presence of noise. The FARDIN, which has been developed and validated by Mahdavi, et al., [20] is based on the study of Heidari, et al., [21] and is calculated in terms of the SNR needed for reaching $50 \%$ correct recognition of Persian monosyllabic digits from 1 to 10 , formatted as three unique digit triplet. For FARDIN, using two equivalent lists of digit triplets, noise was presented at $15 \mathrm{~dB}$ SL ( $15 \mathrm{~dB}$ above 
most comfortable level) from a laptop connected to the clinical audiometer via a front calibrated speaker located $1 \mathrm{~m}$ away from the subject. A carrier phrase ("say") is heard $250 \mathrm{~ms}$ before each triplet. In each triplet, the inter-digit and inter-item gaps are set for $500 \mathrm{~ms}$ and $5 \mathrm{~s}$, respectively. The SNR was changed from $+5 \mathrm{~dB}$ to $-16 \mathrm{~dB}$ in 3-dB steps. Finally, by using the Spearman-Karber equation, the SNR for reaching 50\% correct recognition of nine Persian monosyllabic digits in background noise was calculated.

In addition, to determine the specific effect of hearing aid amplification on the performance of subjects during the FARDIN test for each group, the amplification benefit was defined based on the following equation:

\section{Amplification benefit $=$ Aided SNR - Unaided SNR .}

Thus, a positive value (in $\mathrm{dB}$ ) indicates that the subject's performance in the DIN test (SNR) was higher in the aided condition than in the unaided condition. A negative or zero value demonstrates that SNR was equal or lower in the unaided condition than in the aided condition.

\section{Statistical analysis}

In this study, we used IBM SPSS ver. 24.0 (IBM Corp., Armonk, NY, USA) to analyze the study data. Descriptive statistical parameters, such as the mean and standard deviation (SD) of the SNR results, were considered.

Data distribution was compared by using KolmogorovSmirnov test and the normal distribution was not rejected for all data ( $p$ value ranged from 0.15 to 0.2 for all data). Because of the normal distribution, we used the paired samples t-test for each group to assess differences of investigated variables between tested conditions. The independent t-test was used to compare the study groups. All statistical analyzes were performed at the 0.05 level. In addition, the Pearson correlation coefficient test was performed to evaluate the probable correlation between results.

\section{Results}

\section{SNR}

Under unaided and aided conditions, the SNR ranges of the moderate and severe SNHL groups were from -7.20 to -0.50 versus -5.50 to -0.20 and from -7.80 to -4.20 versus -6.20 to -0.80 , respectively (Table 1 ). For both moderate and severe SNHL groups, shown in Table 1, the mean SNR of the unaided condition is higher (worse) than the aided condition (-4.66 \pm 1.77 vs. $-5.98 \pm 1.15 \mathrm{~dB}$ for the moderate SNHL group and $-2.32 \pm 1.59$ vs. $-3.40 \pm 1.44 \mathrm{~dB}$ for the severe SNHL group).
Table 1. The unaided and aided SNR for each group

\begin{tabular}{cccc}
\hline \multirow{2}{*}{ Groups } & \multicolumn{3}{c}{ SNR $(\mathrm{dB})$} \\
\cline { 2 - 4 } & Minimum & Maximum & Mean \pm SD \\
\hline G1 (moderate SNHL group) & & & \\
Unaided condition & -7.20 & -0.50 & $-4.66 \pm 1.77$ \\
Aided condition & -7.80 & -4.20 & $-5.98 \pm 1.15$ \\
G2 (severe SNHL group) & & & \\
Unaided condition & -5.50 & -0.20 & $-2.32 \pm 1.59$ \\
Aided condition & -6.20 & -0.80 & $-3.40 \pm 1.44$ \\
\hline
\end{tabular}

SNHL: sensorineural hearing loss, SNR: signal-to-noise ratio

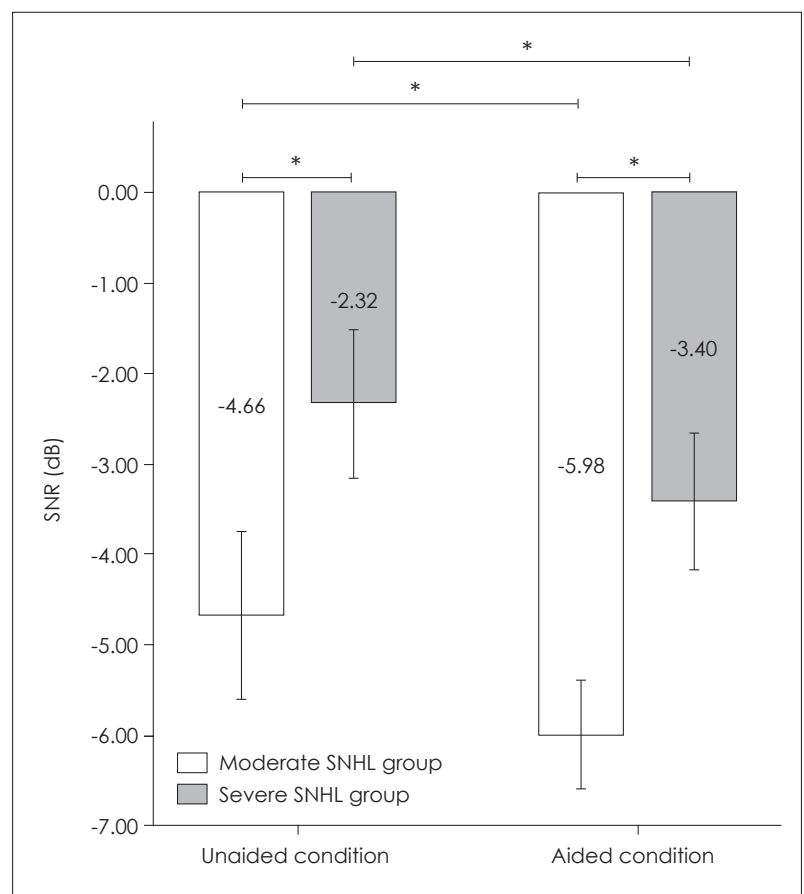

Fig. 2. Comparison of unaided and aided SNR results in the moderate and severe SNHL groups. Error bars indicate standard deviation. ${ }^{*} p<0.001$, significant differences between intra- and intergroup results, respectively. SNR: signal-to-noise ratio, SNHL: sensorineural hearing loss.

Using the paired samples t-test, the SNR difference observed between unaided and aided conditions was statistically significant for both groups $\left(\mathrm{t}_{(16)}=6.20, p<0.001\right.$ and $\mathrm{t}_{(16)}=4.77, p<0.001$ for moderate and severe SNHL groups, respectively) (Fig. 2).

The mean SNR for the moderate SNHL group is lower (better) than that of the severe SNHL group in both unaided $(-4.66 \pm 1.77$ vs. $-2.32 \pm 1.59$, respectively) and aided conditions $(-5.98 \pm 1.5 \mathrm{~dB}$ vs. $-3.40 \pm 1.44 \mathrm{~dB}$, respectively). Using the independent t-test, this observed difference between the tested groups is statistically significant for both conditions $\left(\mathrm{t}_{(32)}=\right.$ $-4.03, p<0.001$ and $\mathrm{t}_{(32)}=-5.74, p<0.001$ for unaided and aided conditions, respectively) (Fig. 2). 


\section{Amplification benefit}

The mean amplification benefit was not statistically significant when comparing the groups ( $p=0.221)$ (Fig. 3). All cases had positive values, indicating better performance in the aided condition than in the unaided condition, except for one participant (number 29 in Fig. 4) that performed approximately

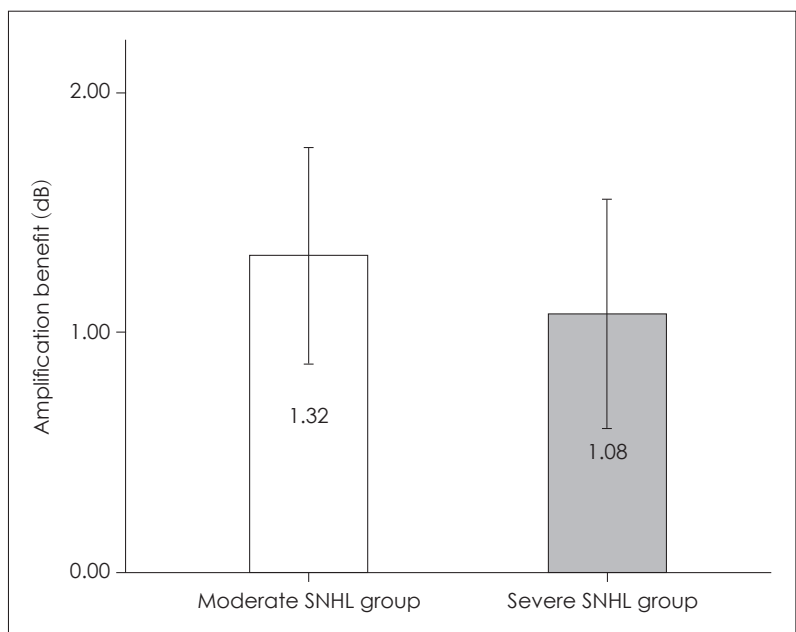

Fig. 3. Amplification benefit of signal-to-noise ratio for moderate and severe SNHL groups. Error bars indicate standard deviation. SNHL: sensorineural hearing loss.

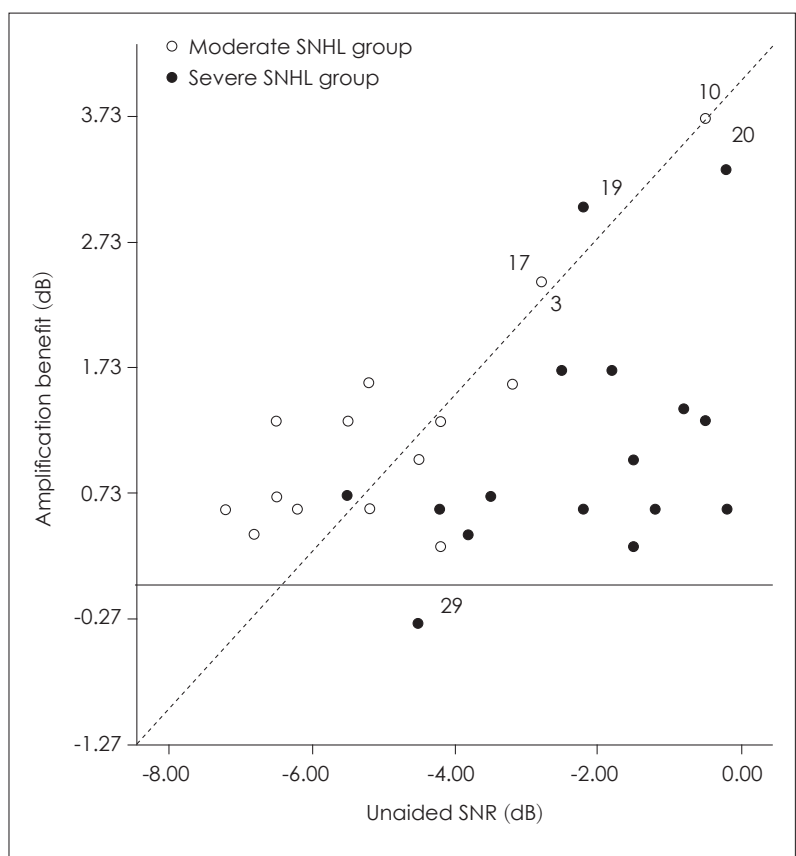

Fig. 4. Amplification benefit of SNR in comparison with the SNR in the unaided condition for moderate and severe SNHL groups (dotted line is a diagonal reference line, which indicates no difference between test conditions when dots are over it, and horizontal solid line shows $0 \mathrm{~dB}$, which means no amplification benefit). Cases with a higher amplification benefit are shown by their own case numbers $(3,10,17,19$, and 20). Case number 29 performed approximately equally well in both conditions. SNR: signal-to-noise ratio, SNHL: sensorineural hearing loss. equally well in both conditions. However, the necessary audibility was provided by the BUMS hearing aid fitting protocol based on the calibrated version of the Ling- 6 sounds for the level of $30 \mathrm{~dB}$ HL.

There were five cases, numbers $3,10,17,19$, and 20 in Fig. 4, who benefited by more than $2 \mathrm{~dB}$ when wearing the fitted hearing aid. Case numbers 3, 10, 17 were from moderate SNHL group (mean PTA $\pm \mathrm{SD}=47.22 \pm 3.47 \mathrm{~dB} \mathrm{HL}$ ) and case numbers 19 and 20 were from severe SNHL group (mean $\mathrm{PTA} \pm \mathrm{SD}=65.83 \pm 1.18 \mathrm{~dB}$ HL) . Whereas, there was no benefits from hearing aid amplification for case number 29. She was from severe SNHL group with flat hearing loss configuration (PTA=67 dB HL). However, by using the BUMS hearing aid fitting protocol, the equal audibility for soft sounds was provided for all these above cases.

\section{Unaided and aided SNR correlation}

The pooled SNR results revealed a significant strong correlation between unaided SNR and aided SNR ( $\mathrm{r}=0.90, p<0.001)$. For each group (G1 and G2), there was a significant strong positive correlation between unaided SNR and aided SNR ( $\mathrm{r}=$ $0.91, p<0.001$ and $\mathrm{r}=0.82, p<0.001$, respectively). To facilitate comparison, the unaided SNR was plotted on the horizontal axis (x-axis), and the aided condition, which may help improve performance in all subjects, was plotted on the vertical

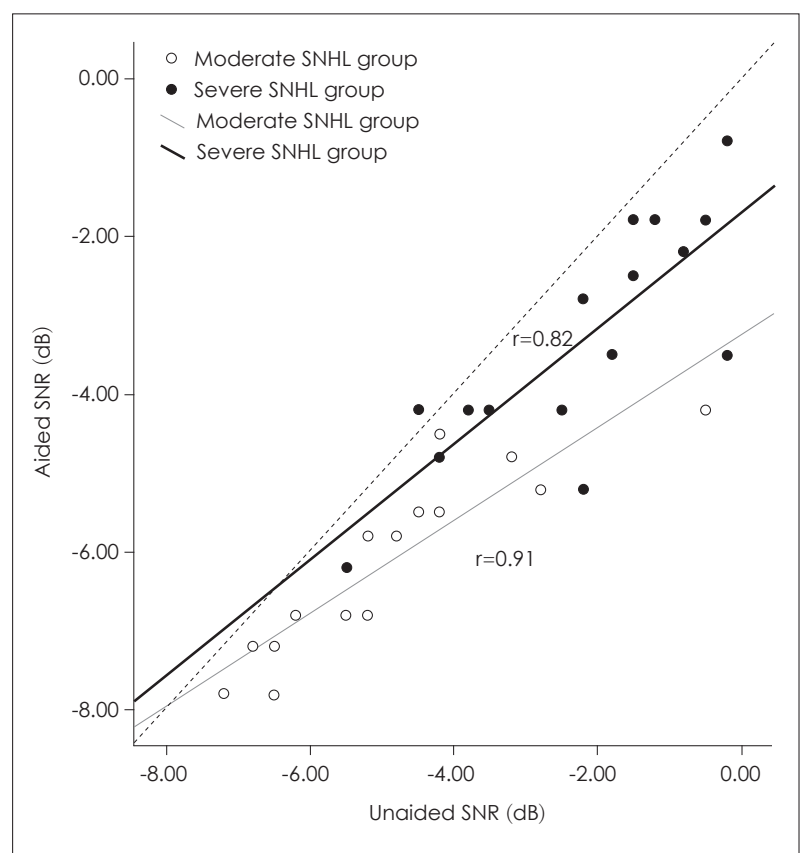

Fig. 5. Scatterplots showing the SNR (in dB) for individual participants between two test conditions. Points below the diagonal reference line (dotted line) reflect better performance with a hearing aid. The best fit line for the moderate and severe SNHL groups is shown by the grey and black line, respectively. SNR: signal-tonoise ratio, SNHL: sensorineural hearing loss. 
Table 2. Pearson correlation between HTLs of each frequency and average of all HTLs (250 through $8,000 \mathrm{~Hz}$ ) and SNR in unaided and aided conditions

\begin{tabular}{lcrccc}
\hline & \multicolumn{2}{l}{ Unaided SNR condition } & & \multicolumn{2}{c}{ Aided SNR condition } \\
\cline { 2 - 3 } \cline { 5 - 6 } HTL $(\mathrm{Hz})$ & $\begin{array}{c}\text { Pearson } \\
\text { correlation }\end{array}$ & p value & & $\begin{array}{c}\text { Pearson } \\
\text { correlation }\end{array}$ & p value \\
\hline 250 & 0.52 & 0.002 & & 0.64 & $<0.001$ \\
500 & 0.59 & $<0.001$ & & 0.70 & $<0.001$ \\
1,000 & 0.51 & 0.002 & & 0.66 & $<0.001$ \\
2,000 & 0.51 & 0.002 & & 0.67 & $<0.001$ \\
4,000 & 0.61 & $<0.001$ & & 0.70 & $<0.001$ \\
8,000 & 0.58 & $<0.001$ & & 0.66 & $<0.001$ \\
Average & 0.59 & $<0.001$ & & 0.71 & $<0.001$ \\
\hline
\end{tabular}

HTL: hearing threshold level, SNR: signal-to-noise ratio

axis (y-axis) (Fig. 5). All data points were below the diagonal reference line, indicating that the hearing aid improved subjects' performance over that in the unaided condition, except for case number 29 (Fig. 5).

The pooled Pearson correlation coefficient demonstrated a significant positive correlation between the hearing threshold levels (HTLs) of all frequencies and the average of all HTLs and SNR in both unaided and aided conditions (Table 2).

\section{Discussion}

This study demonstrates that, firstly, there is a significant difference in subjects' speech recognition between the clinical setup condition, which is evaluated using an audiometer in a free field, and hearing aid amplification condition, which uses a hearing aid fit based on NAL-NL2-assessed hearing loss configuration. Secondly, in order to have the same speech intelligibility in the presence of noise under both free field and hearing aid amplification conditions, listeners with severe SNHL need higher SNRs than listeners with moderate SNHL. It seems that the amplification of sounds based on an evidence-based fitting rationale (e.g., NAL-NL2) helps improve the speech recognition of hearing-impaired people in the presence of noise- - something that cannot be realized exactly using the conventional clinical setup (audiometer and free-field test). The conventional free-field testing condition just measures the subject's performance for speech recognition in the presence of noise clinically rather than predicting the amplification benefit due to the use of a hearing aid with a gain frequency response shaped based on hearing loss configuration. To determine the exact amplification benefit based on the gain frequency response shaping or any digital signal processing, the hearing aid has to be fit based on the intended setup, and the hearing-impaired person should be tested using the fitted hearing aid.

Consistent with the finding of a more recent study [13], which applied a similar setup for patients with active implant hearing aids using a word recognition test, the strong correlation between unaided and aided SNRs observed this study suggests that the speech recognition of the patient measured through an audiometer for the typical free-field frequency response in clinical conditions can just be used as a general marker of his or her ability to assess the intelligibility of speech in noise. It cannot be used as an indicator of the benefit of wearing a hearing aid with an individualized gain frequency response in accordance with the patient's hearing loss configuration.

Moreover, this is the first study that investigates the results of the Persian version of the DIN test among hearing-impaired patients. Compared with the mean SNR reported for healthy people $(-9.5 \mathrm{~dB})$ [21], the SNR for people with moderate and severe SNHL in the present study are significantly higher (by about $5 \mathrm{~dB}$ and $7 \mathrm{~dB}$, respectively). A greater increase is seen in patients with more severe SNHL. It seems that having higher hearing thresholds implies less about performance on the speech-in-noise test, a finding that is consistent with previous studies $[1,9]$. The results of the present study are consistent with the results of a large-scale current study that found a significant correlation between the results of the DIN test and degree of hearing loss [10]. The fact that there is an association between the degree of hearing loss and speech intelligibility in the presence of noise suggests that the extraction of speech signals from background noise strongly depends on the mechanism(s) of SNHL [1]. Although part of the problem of a hearing-impaired patient's performance in the presence of noise could be because of the inability to hear speech signals (especially high frequency cues), it seems that the main problem should be explained by other most important causes, i.e., hearing loss desensitization. As shown in this study, the audibility provided by both the hearing aid (with a suitable amplification frequency response, which is based on the latest model of the speech intelligibility index to provide maximum speech intelligibility) not only does not result in a much greater benefit regarding speech recognition in noise for most of the people with severe SNHL, but also for those with less (moderate) SNHL. On average, the audibility showed a $1 \mathrm{~dB}$ improvement in SNR in both the moderate and severe SNHL groups. However, more improvement was observed in some cases of both moderate (case numbers 3, 10, and 17) and severe SNHL (case number 19 and 20) at an individual level. As it has been proven [22], in addition to the auditory peripheral status, the cognitive ability including memory and attention is involved in speech recognition in noise as well, especially while wearing hearing aid. Although we controlled the potential effects of cognitive status using the MMSE, the more benefit observed for case numbers $3,10,17,19$, and 20 might be associated with their cognitive 
performance, a most important factor playing a major role that its variability is needed to be evaluated. Therefore, more benefit observed for case numbers $3,10,17,19$, and 20 and no benefit from hearing aid amplification for case number 29 might be associated with the different cognitive performance among subjects, a variable that should be considered in future researches.

We only included experienced hearing aid users (full day users with at least 2 years of experience of using hearing aid amplification for both groups) in this study to rule out the role of amplification acclimatization [12], as well as the skillful extraction of speech signals from noise and professional usage for intelligibility based on hearing aid (or amplification) experience, in order to control influencing factors on speech performance in the presence of noise. According to a theory explaining acclimatization (refocus of auditory attention), background noise has a distracting effect (informational masking) on speech recognition [23]. More recently, it has been shown that experienced hearing aid users have faster (better) processing time for speech intelligibility in the presence of noise than inexperienced users [24,25]. It seems that experienced hearing aid users, by acclimatization, can ignore the distraction effect. In other words, following acclimatization, they are able to "tune out" aversive sounds and noises. Consequently, they obtain additional benefit from amplification, as well as hearing aid, technologies. In addition, it has been shown that digital noise reduction technology improves the tracking speed performance of hearing aid users in a dual-task paradigm of speech intelligibility [26]. This would suggest hearing aid experience could provide fast speech comprehension in the presence of noise. Therefore, to investigate the current study results among inexperienced hearing aid users, a future study should be conducted.

In conclusion, speech recognition in hearing-impaired patients, which deteriorates when the degree of hearing loss increases, can be realized by using hearing aids fit based on an evidence-based fitting rationale (NAL-NL2) rather than by measuring it using free-field audiometry measurement that is typically utilized in a routine clinic setup. In order to determine the amplification benefit of hearing aids, we need to measure speech recognition in noise when the hearing aid is fitted based on amplification targets according to the patients' hearing loss configuration.

\section{Acknowledgments}

None

\section{Conflicts of interest}

The authors have no financial conflicts of interest.

\section{Author Contributions}

Conceptualization: Mina Aghasoleimani and Hamid Jalilvand.
Data Curation: Mina Aghasoleimani and Roghayeh Ahmadi. Formal analysis: Mina Aghasoleimani. Funding acquisition: Hamid Jalilvand and Mina Aghasoleimani. Investigation: Mina Aghasoleimani. Methodology: Hamid Jalilvand and Mohammad Ebrahim Mahdavi. Project administration: Hamid Jalilvand. Resources: Hamid Jalilvand. Supervision: Hamid Jalilvand. Validation: Hamid Jalilvand and Mohammad Ebrahim Mahdavi. Visualization: Hamid Jalilvand. Writing — original draft: Hamid Jalilvand and Mina Aghasoleimani. Writing - review \& editing: all authors. Approval of final manuscript: all authors.

\section{ORCID iDs}

Mina Aghasoleimani https://orcid.org/0000-0003-0539-8794

Hamid Jalilvand https://orcid.org/0000-0002-2351-5918

Mohammad Ebrahim Mahdavi

https://orcid.org/0000-0002-7589-7065

Roghayeh Ahmadi https://orcid.org/0000-0003-2165-2051

\section{REFERENCES}

1) Ching TY, Dillon H. A brief overview of factors affecting speech intelligibility of people with hearing loss: implications for amplification. Am J Audiol 2013;22:306-9.

2) Moore BC. A review of the perceptual effects of hearing loss for frequencies above $3 \mathrm{kHz}$. Int J Audiol 2016;55:707-14.

3) Grant KW, Walden TC. Understanding excessive SNR loss in hearing-impaired listeners. J Am Acad Audiol 2013;24:258-73.

4) Houtgast T, Festen JM. On the auditory and cognitive functions that may explain an individual's elevation of the speech reception threshold in noise. Int J Audiol 2008;47:287-95.

5) Taylor BB. Speech-in-noise tests: how and why to include them in your basic test battery. Hear J 2003;56:40-3.

6) Smits C, Kapteyn TS, Houtgast T. Development and validation of an automatic speech-in-noise screening test by telephone. Int J Audiol 2004;43:15-28.

7) Wilson RH, Burks CA, Weakley DG. Word recognition of digit triplets and monosyllabic words in multitalker babble by listeners with sensorineural hearing loss. J Am Acad Audiol 2006;17:385-97.

8) Smits C, Theo Goverts S, Festen JM. The digits-in-noise test: assessing auditory speech recognition abilities in noise. J Acoust Soc Am 2013;133:1693-706.

9) Jansen S, Luts H, Dejonckere P, van Wieringen A, Wouters J. Efficient hearing screening in noise-exposed listeners using the digit triplet test. Ear Hear 2013;34:773-8.

10) Koole A, Nagtegaal AP, Homans NC, Hofman A, Baatenburg de Jong RJ, Goedegebure A. Using the digits-in-noise test to estimate age-related hearing loss. Ear Hear 2016;37:508-13.

11) Moore DR, Edmondson-Jones M, Dawes P, Fortnum H, McCormack A, Pierzycki RH, et al. Relation between speech-in-noise threshold, hearing loss and cognition from 40-69 years of age. PLoS One 2014; 9:e107720.

12) Dillon H. Hearing aids. 2 ed. New York: Thieme;2012.

13) McRackan TR, Ahlstrom JB, Clinkscales WB, Meyer TA, Dubno JR. Clinical implications of word recognition differences in earphone and aided conditions. Otol Neurotol 2016;37:1475-81.

14) Schlauch RS, Nelson P. Puretone evaluation. In: Handbook of clinical audiology (eds. Katz J, Chasin M, English K, Hood LJ, Tillery KL), 7th ed. Philadelphia: Wolters Kluwer;2015. p.29-47.

15) Ansari NN, Naghdi S, Hasson S, Valizadeh L, Jalaie S. Validation of a Mini-Mental State Examination (MMSE) for the Persian population: a pilot study. Appl Neuropsychol 2010;17:190-5.

16) Scollie S, Glista D, Tenhaaf J, Dunn A, Malandrino A, Keene K, et al. Stimuli and normative data for detection of Ling-6 sounds in hear- 
ing level. Am J Audiol 2012;21:232-41.

17) Aghsoleimani M, Jalilvand H, Mahdavi ME, Nazeri AR, Kamali M. The acceptable noise level benefit from directionality for listeners with severe hearing loss. Clin Exp Otorhinolaryngol 2018;11:166-73.

18) Ahmadi R, Jalilvand H, Mahdavi ME, Ahmadi F, Baghban ARA. The effects of hearing aid digital noise reduction and directionality on acceptable noise level. Clin Exp Otorhinolaryngol 2018;11:267-74.

19) The American National Standards Institute. American National Standard: maximum permissible ambient noise levels for audiometric test rooms. ANSI/ASA S3.1-1999 (R2018). Melville, NY: Acoustical Society of America;2008.

20) Mahdavi ME, Pourbakht A, Parand A, Jalaie S, Rezaeian M, Moradiju E. Auditory recognition of words and digits in multitalker babble in learning-disabled children with dichotic listening deficit. Iran Red Crescent Med J 2017;19:e42817.

21) Heidari M, Mahdavi ME, Heidari F, Baghban AA. Auditory recognition of Persian digits in multi-talker babble noise: a preliminary study.
Aud Vest Res 2015;24:134-40.

22) Dryden A, Allen HA, Henshaw H, Heinrich A. The association between cognitive performance and speech-in-noise perception for adult listeners: a systematic literature review and meta-analysis. Trends Hear 2017;21:2331216517744675.

23) Dawes P, Munro KJ. Auditory distraction and acclimatization to hearing aids. Ear Hear 2017;38:174-83.

24) Habicht J, Kollmeier B, Neher T. Are experienced hearing aid users faster at grasping the meaning of a sentence than inexperienced users? An eye-tracking study. Trends Hear 2016;20:2331216516660966.

25) Wendt D, Kollmeier B, Brand T. How hearing impairment affects sentence comprehension: using eye fixations to investigate the duration of speech processing. Trends Hear 2015;19:2331216515584149.

26) Desjardins JL, Doherty KA. The effect of hearing aid noise reduction on listening effort in hearing-impaired adults. Ear Hear 2014;35:60010. 